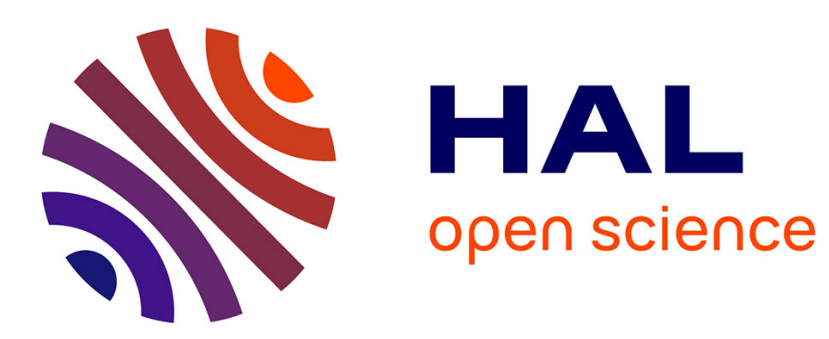

\title{
New exact relations for easy recovery of steady wave profiles from bottom pressure measurements.
}

Didier Clamond

\section{To cite this version:}

Didier Clamond. New exact relations for easy recovery of steady wave profiles from bottom pressure measurements.. Journal of Fluid Mechanics, 2013, 726, 10.1017/jfm.2013.253 . hal-01302712

\section{HAL Id: hal-01302712 \\ https://hal.science/hal-01302712}

Submitted on 20 Dec 2018

HAL is a multi-disciplinary open access archive for the deposit and dissemination of scientific research documents, whether they are published or not. The documents may come from teaching and research institutions in France or abroad, or from public or private research centers.
L'archive ouverte pluridisciplinaire HAL, est destinée au dépôt et à la diffusion de documents scientifiques de niveau recherche, publiés ou non, émanant des établissements d'enseignement et de recherche français ou étrangers, des laboratoires publics ou privés. 


\title{
New exact relations for easy recovery of steady wave profiles from bottom pressure measurements
}

\author{
Didier Clamond $\dagger$ \\ Laboratoire J. A. Dieudonné, Université de Nice - Sophia Antipolis, Parc Valrose, \\ 06108 Nice CEDEX 2, France
}

This paper is about the determination of a free surface wave profile from a given pressure distribution at the bottom. For a two-dimensional irrotational steady surface water wave propagating over a flat horizontal bed, this problem is solved analytically in an implicit form. Explicit solutions can be easily obtained numerically via fixed point iterations, whose convergence is outlined mathematically and numerically. This new surface reconstruction procedure does not involve the resolution of a differential or an integral equation, i.e. the surface is given by algebraic local relations. Thus, this formulation permits the free surface recovery in a simpler and more efficient way than previous methods.

Key words: surface gravity waves, waves/free-surface flows

\section{Introduction}

Within the framework of incompressible, homogeneous and inviscid flow modelling of water waves, the pressure plays an important role in establishing various qualitative properties of travelling waves. The pressure is essential in quantitative studies because often in practice the elevation of a surface water wave is determined from pressure data obtained at the sea bed (see, e.g., Tsai et al. 2005). Indeed, measuring a free surface wave motion without intrusive probes is of great importance for practical applications, and one way to achieve this is to use pressure gauges at the sea bed. From these pressure measurements, the question is then how can one reconstruct the free surface. This problem has long been of interest and has recently attracted attention (e.g. Constantin 2012; Deconinck, Oliveras \& Vasan 2012; Oliveras et al. 2012; Clamond \& Constantin 2013).

The first approaches to reconstructing the free surface from pressure measurements consisted of assuming that the hydrostatic approximation is sufficiently accurate. However, for waves of moderate amplitude, prediction errors are significant (Bishop \& Donelan 1987). These inaccuracies are due to the fact that the hydrostatic approximation ignores even linear wave effects. Taking into account the wave effect to the linear approximation, one can derive a better approximation (Escher \& Schlurmann 2008), but the benefit is limited for waves of moderate amplitude (Tsai et al. 2005).

$\dagger$ Email address for correspondence: didierc@unice.fr 
These considerations motivated the quest for a reconstruction formula that accounts for all nonlinear effects and that is thus applicable to waves of moderate and large amplitude. Nonlinear non-local equations relating the dynamic pressure on the bed and the wave profile were obtained without approximation from the governing equations (Constantin 2012; Oliveras et al. 2012).

Very recently, other exact tractable relations have been obtained (Clamond \& Constantin 2013), allowing mathematical analysis and straightforward numerical procedures for deriving the free surface from the pressure at the bed. This surface reconstruction method requires the resolution of an ordinary differential equation. Although the numerical resolution of this differential equation is numerically easy, it is actually possible to solve it analytically in an implicit form, thus providing an even simpler reconstruction procedure. The purpose of the present paper is to show how and to provide mathematical insights of the efficiency of this new approach, as well as some numerical evidence.

This paper is organized as follows. In $\S 2$, the hypothesis and notation are introduced for steady irrotational surface waves on constant depth. In order to make the present paper self-contained, the main results of Clamond \& Constantin (2013) are given in $\S 3$. A new, purely local, expression for the free surface is then derived in $\S 4$. It requires the introduction of a new holomorphic which can be easily calculated, as demonstrated in $\S 5$. The convergence properties of the new formula for the surface are outlined in $\S 6$. Finally, numerical examples are provided in $\S 7$.

\section{Hypothesis and notation}

The physical hypothesis and most mathematical notation are identical to those of Clamond \& Constantin (2013). For the sake of completeness, they are briefly recalled here.

We consider a steady irrotational wave motion due to a surface gravity wave of permanent form. Let $(x, y)$ be a Cartesian coordinate system moving with the wave, $x$ being the horizontal coordinate and $y$ the upward vertical coordinate. The wave is $(2 \pi / k)$-periodic in the $x$ direction, solitary waves corresponding to the limiting case $k \rightarrow 0$. Here $y=-d, y=\eta(x)$ and $y=0$ denote the equations of the horizontal impermeable bottom, of the impermeable free surface and of the mean water level, respectively. The latter equation expresses the fact that $\langle\eta\rangle=0$ for the smooth $(2 \pi / k)$-periodic wave profile $\eta$, where $\langle\cdot\rangle$ is the Eulerian average operator over one wavelength, i.e.

$$
\langle\eta\rangle \equiv \frac{k}{2 \pi} \int_{-\pi / k}^{\pi / k} \eta(x) \mathrm{d} x=0 .
$$

Let $u, v, \phi$ and $\psi$ be the horizontal velocity, the vertical velocity, the velocity potential and the stream function, respectively, such that $u=\phi_{x}=\psi_{y}$ and $v=\phi_{y}=$ $-\psi_{x}$. Also let the complex potential $f=\phi+\mathrm{i} \psi$ and the complex velocity $w=u-\mathrm{i} v$ (with $\mathrm{i}^{2}=-1$ ) be holomorphic functions of the complex abscissa $z=x+\mathrm{i} y$, i.e. $f=f(z)$ and $w=\mathrm{d} f / \mathrm{d} z$. The mass conservation and the irrotational character of the flow are identically fulfilled with the requirement that $f$ and $w$ are holomorphic functions throughout the fluid domain. The flow being steady, the bottom and surface boundaries being impermeable, the stream function $\psi=\operatorname{Im}\{f\}$ is constant at $y=-d$ and at $y=\eta$. Thus, $v_{s}=\eta_{x} u_{s}$ (with $\eta_{x}=\mathrm{d} \eta / \mathrm{d} x$ ) and $v_{b}=0$, where subscripts ' $s$ ' and ' $b$ ' denote the restrictions at the free surface and at the bed, respectively. 
The momentum equation can be expressed by means of the Bernoulli condition

$$
2 p+2 g y+u^{2}+v^{2}=B, \quad x \in \mathbb{R},-d \leqslant y \leqslant \eta,
$$

for some Bernoulli constant $B$, and where $p=p(x, y)$ is the pressure divided by the (constant) density. At the free surface, the surface tensions are neglected and the pressure is zero, i.e. $p_{s}=0$. From (2.1) and (2.2), we get relations for the Bernoulli constant

$$
B=\left\langle u_{s}^{2}+v_{s}^{2}\right\rangle=\left\langle u_{b}^{2}\right\rangle>0,
$$

the second equality in (2.3) being a classical result (see, e.g. Longuet-Higgins 1975; Clamond \& Constantin 2013). The relations (2.2) and (2.3) yield in turn

$$
\langle\mathfrak{p}\rangle=g d,
$$

where $\mathfrak{p}(x) \equiv p(x,-d)=p_{b}(x)$ is the normalized pressure at the bed.

We also define the wave phase velocity $c$ such that $c=-\left\langle u_{b}\right\rangle$, so that the wave travels with phase speed $c$ in the frame of reference where the mean horizontal velocity is zero at the bed, and where $c>0$ if the wave travels toward the increasing $x$ direction. For smooth waves, the inequality $u / c<0$ occurs everywhere in the fluid and at the free surface, a stagnation point (where $u=v=0$ ) being possible only at the angular crests of the highest waves. Note that $B=c^{2}$ in deep water $(d \rightarrow \infty)$ and for solitary waves $(k \rightarrow 0)$, but $B \neq c^{2}$ in general.

\section{Principle of the surface recovery}

Following Clamond \& Constantin (2013), let be the holomorphic function $\mathfrak{P}$ such that

$$
\begin{aligned}
\mathfrak{P}(z) & \equiv \frac{1}{2} B+g d-\frac{1}{2} w^{2}(z) \\
& =\frac{1}{2} B+g d-\frac{1}{2}\left(u^{2}-v^{2}\right)+\mathrm{i} u v,
\end{aligned}
$$

having thus restriction at the flat bed $y=-d$ with zero imaginary part and real part $\mathfrak{p}$, i.e. $\mathfrak{p}(x)=\mathfrak{P}_{b}=\mathfrak{P}(x-\mathrm{i} d)=g d+\left(B-u_{b}^{2}\right) / 2$, thus $\mathfrak{P}(z)=\mathfrak{p}(z+\mathrm{i} d)$. Here $p$ coincides with the real function $\operatorname{Re}\{\mathfrak{P}\}$ only on $y=-d$ because $p$ is not a harmonic function (Constantin 2006; Constantin \& Strauss 2010).

Using the surface impermeability and (2.2) on the free surface $y=\eta(x)$, where $p=0$ and $v_{s}=u_{s} \eta_{x}$, we have

$$
\begin{aligned}
\left(u_{s}-\mathrm{i} v_{s}\right)^{2} & =\left(1-\mathrm{i} \eta_{x}\right)^{2} u_{s}^{2}=\left(1+\eta_{x}^{2}\right) u_{s}^{2}\left(1-\mathrm{i} \eta_{x}\right) /\left(1+\mathrm{i} \eta_{x}\right) \\
& =\left(u_{s}^{2}+v_{s}^{2}\right)\left(1-\mathrm{i} \eta_{x}\right) /\left(1+\mathrm{i} \eta_{x}\right)=(B-2 g \eta)\left(1-\mathrm{i} \eta_{x}\right) /\left(1+\mathrm{i} \eta_{x}\right) .
\end{aligned}
$$

Multiplying this relation by $\left(1+\mathrm{i} \eta_{x}\right)$ and using (3.1), we obtain at once

$$
g \eta\left(1-\mathrm{i} \eta_{x}\right)+\mathrm{i} B \eta_{x}=\left(\mathfrak{P}_{s}-g d\right)\left(1+\mathrm{i} \eta_{x}\right),
$$

with $\mathfrak{P}_{s}=\mathfrak{P}\left(z_{s}\right)=\mathfrak{P}(x+\mathrm{i} \eta(x))$. The real and imaginary parts of (3.3) give the two equations

$$
\begin{aligned}
g \eta & =\operatorname{Re}\left\{\mathfrak{P}_{s}\right\}-g d-\eta_{x} \operatorname{Im}\left\{\mathfrak{P}_{s}\right\}=\frac{1}{2}\left(B-u_{s}^{2}-v_{s}^{2}\right), \\
(B-g \eta) \eta_{x} & =\left(\operatorname{Re}\left\{\mathfrak{P}_{s}\right\}-g d\right) \eta_{x}+\operatorname{Im}\left\{\mathfrak{P}_{s}\right\}=\frac{1}{2}\left(B+u_{s}^{2}+v_{s}^{2}\right) \eta_{x}
\end{aligned}
$$

where the second equalities are obtained using (3.1) and $v_{s}=\eta_{x} u_{s}$. 
The wave amplitude $a$ is obtained by evaluating (3.4) at the wave crest located at $x=0$ (where $\eta_{x}=0$ and $\eta=a \geqslant 0$ ) leading to the implicit equation

$$
a=g^{-1} \operatorname{Re}\{\mathfrak{P}(\mathrm{i} a)\}-d .
$$

Similarly, at the wave trough located at $x=\pi / k$ (where $\eta_{x}=0$ and $\eta=-b \leqslant 0, a+b$ being thus the total wave height), we have the corresponding implicit equation

$$
b=d-g^{-1} \operatorname{Re}\{\mathfrak{P}(\pi / k-\mathrm{i} b)\} .
$$

The crest height $a$ is obtained as the unique solution to (3.6), as proven by Clamond $\&$ Constantin (2013). The convergence of (3.7) is discussed in $\S 6$ below.

With the wave amplitude $a$ determined by solving (3.6) iteratively, $\eta$ is subsequently obtained by re-expressing (3.5) as the ordinary differential equation

$$
\eta_{x}=\operatorname{Im}\left\{\mathfrak{P}_{s}\right\} /\left(B-g \eta-\operatorname{Re}\left\{\mathfrak{P}_{s}\right\}+g d\right),
$$

with initial data $\eta(0)=a$. The right-hand side of (3.8) being smooth, the solution can be obtained by a standard iterative procedure, as proven and illustrated by Clamond \& Constantin (2013). It is however possible to derive a local simpler expression which does not require the resolution of the differential equation. In other words, the differential equation (3.8) can be solved analytically in an implicit form, leading to an algebraic equation for $\eta$, as shown in the next section.

The surface reconstruction procedure described above is mathematically valid for all Stokes waves, as proven by Clamond \& Constantin (2013). Although the procedure is likely also valid for all regular waves, a rigourous mathematical proof is lacking. Note that, by 'Stokes waves', Constantin (2006), Constantin \& Strauss (2010) and Clamond \& Constantin (2013) mean all regular periodic symmetric waves with one crest and one trough per wavelength. According to this definition, solitary waves are a limiting case of Stokes waves. This is not the case with the more conventional definition, where 'Stokes waves' are approximations derived from a Poincaré-Lindstedt perturbation scheme that breaks down in shallow water and thus cannot describe solitary waves.

\section{New equations for the surface recovery}

Let $\mathfrak{Q}$ be yet another holomorphic function such that

$$
\mathfrak{Q}(z) \equiv \int_{z_{0}}^{z}\left[\mathfrak{P}\left(z^{\prime}\right)-g d\right] \mathrm{d} z^{\prime}=\int_{z_{0}}^{z} \frac{1}{2}\left[B-w\left(z^{\prime}\right)^{2}\right] \mathrm{d} z^{\prime},
$$

where $z_{0}$ is an arbitrary constant. Taking $z_{0}$ at the origin of the free surface (i.e. $x=0$, $y=a$, thus $z_{0}=\mathrm{i} a$ ) and choosing the free surface as integration path, the definition (4.1) yields

$$
\mathfrak{Q}_{s}(x)=\int_{0}^{x}\left[\mathfrak{P}\left(x^{\prime}+\mathrm{i} \eta\left(x^{\prime}\right)\right)-g d\right]\left[1+\mathrm{i} \eta_{x}\left(x^{\prime}\right)\right] \mathrm{d} x^{\prime},
$$

thus, substituting (3.3) and after some elementary algebra,

$$
\mathfrak{Q}_{s}(x)=\int_{0}^{x} g \eta\left(x^{\prime}\right) \mathrm{d} x^{\prime}+\mathrm{i}[\eta(x)-a]\left[B-\frac{1}{2} g a-\frac{1}{2} g \eta(x)\right] .
$$

It is worth noting that the choice of $z_{0}$ in the definition of $\mathfrak{Q}$ is obviously unessential, with $z_{0}=\mathrm{i} a$ being chosen above for convenience. In particular, if $z_{0}$ is any point at the 
free surface (i.e. $z_{0}=x_{0}+\mathrm{i} \eta_{0}$ with $\eta_{0}=\eta\left(x_{0}\right)$ ), then one obtains a relation similar to (4.3) with $\int_{0}^{x}$ replaced by $\int_{x_{0}}^{x}$ and $a$ replaced by $\eta_{0}$.

The imaginary part of (4.3) provides a quadratic algebraic equation in $\eta$, relating the latter to $\operatorname{Im}\left\{\mathfrak{Q}_{s}\right\}$. Solving this quadratic equation for $\eta$, one immediately obtains

$$
\eta=g^{-1}\left[B-\sqrt{(B-g a)^{2}-2 g \operatorname{Im}\left\{\mathfrak{Q}_{s}\right\}}\right],
$$

where the minus sign in front of the radical is chosen (instead of the plus sign) in order to fulfil the relation $\eta(0)=a$. In (4.4), substituting $\operatorname{Im}\left\{\mathfrak{Q}_{s}\right\}$ obtained from (4.2), we have a local algebraic (i.e. neither differential nor integral) relation defining the free surface $\eta$ because, in practice, the integral in (4.2) can be computed analytically in closed form (see the examples in $\S 5$ ).

The relation (4.4) is an exact solution of (3.8) written in implicit form, as one can easily check directly from the derivative of (4.4) and using (4.2), i.e.

$$
\eta_{x}=\frac{\left[\operatorname{Im}\left\{\mathfrak{Q}_{s}\right\}\right]_{x}}{\sqrt{(B-g a)^{2}-2 g \operatorname{Im}\left\{\mathfrak{Q}_{s}\right\}}}=\frac{\operatorname{Im}\left\{\mathfrak{P}_{s}\right\}+\left(\operatorname{Re}\left\{\mathfrak{P}_{s}\right\}-g d\right) \eta_{x}}{B-g \eta},
$$

then solving (4.5) for $\eta_{x}$, yielding (3.8) precisely.

For practical applications, the relation (4.4) is obviously a major improvement compared with (3.8). Indeed, using (3.8) requires the numerical resolution of a nonlinear differential equation of the form $\eta_{x}=\Phi(x, \eta)$, while with (4.4) we have to solve an equation of the form $\eta=\Psi(x, \eta)$. Thus, the latter gives access to the free surface at a single point $x=x_{0}$ without computing $\eta$ at the neighbouring points, while using (3.8) requires the determination of $\eta$ over the whole period.

Note that analytic (explicit or implicit) solutions are not necessarily useful in practice if they involve hard-to-compute special functions or/and slowly convergent series. This is not the case here since, for practical surface recovery, few elementary functions are involved in (4.4), as shown below.

An equation for the Bernoulli constant is derived averaging the imaginary part of (4.3) and using (2.1)

$$
2 a B=g\left(a^{2}-\left\langle\eta^{2}\right\rangle\right)-2\left\langle\operatorname{Im}\left\{\mathfrak{Q}_{s}\right\}\right\rangle .
$$

This equation for $B$ is simpler than that derived by Clamond \& Constantin (2013). However, the relation (4.6) involves integrations and is therefore non-local. An alternative explicit closed-form expression for $B$ is obtained if both $a$ and $b$ are already known from, respectively, the local relations (3.6) and (3.7). Indeed, from (4.4) written at the trough $(x=\pi / k, \eta=-b)$, one obtains

$$
B=\frac{1}{2} g(a-b)-(a+b)^{-1} \operatorname{Im}\{\mathfrak{Q}(\pi / k-\mathrm{i} b)\},
$$

that is a very simple expression for $B$ once $a, b$ and $\mathfrak{Q}$ are known.

With the pressure at the bottom being known, it is trivial to obtain $\mathfrak{P}$ and subsequently $\mathfrak{Q}$, as indicated in the next section. Once $a, b$ and $\mathfrak{Q}$ have been determined, an explicit expression for $\eta$ can be obtained numerically via fixed-point iterations of (4.4), whose convergence is studied in $\S 6$.

\section{Examples of analytic approximations for the function $\mathfrak{Q}$}

For the recovery of the surface wave profile $\eta$, given the pressure $\mathfrak{p}$ at the seabed, one can proceed as in the two examples below. 


\subsection{Fourier reconstruction}

For periodic waves, the pressure measurements at the bed can be approximated by a $N$ th-order Fourier polynomial and the function $\mathfrak{P}$ is obtained immediately:

$$
\mathfrak{p}(x) \approx g d+\sum_{|n|>0}^{N} \mathfrak{p}_{n} \exp (\mathrm{i} n k x) \Rightarrow \mathfrak{P}(z)=g d+\sum_{|n|>0}^{N} \mathfrak{p}_{n} \frac{\exp (\mathrm{i} n k z)}{\exp (n k d)},
$$

with $\mathfrak{p}_{-n}=\overline{\mathfrak{p}}_{n}$ since $\mathfrak{p}$ is real. The amplitude $a$ and the trough height $b$ are then determined from (3.6) and (3.7). The function $\mathfrak{Q}$ is subsequently easily obtained as

$$
\mathfrak{Q}(z)=\sum_{|n|>0}^{N} \frac{\mathfrak{p}_{n}}{\mathrm{i} n k} \frac{\exp (\mathrm{inkz})-\exp (-n k a)}{\exp (n k d)},
$$

showing that the determination of $\mathfrak{Q}$ from $\mathfrak{p}$ is a trivial matter. Note that the antiderivative, required to determine $\mathfrak{Q}$, has a natural smoothing effect (i.e. the Fourier coefficient of $\mathfrak{Q}$ decay faster than those of $\mathfrak{P}$ ) that is an interesting feature for practical applications. The Bernoulli constant $B$ is then computed from (4.7) and, finally, one obtains the free surface for $-b<\eta<a$ iterating (4.4).

\subsection{Cnoidal-like reconstruction}

The Fourier polynomial approximation (5.1) is not the only possible approximation and it is not suitable in shallow water, especially for solitary waves. Clamond \& Constantin (2013) suggested another simple approximation able to deal with waves in shallow water. It is derived from the shallow-water approximation and involves the Jacobian elliptic functions $\{\mathrm{sn}, \mathrm{cn}, \mathrm{dn}\}$ and the elliptic integrals $K$ and $E$ of parameter $m$ (Abramowitz \& Stegun 1965), i.e.

$$
\mathfrak{p}(x) \approx g d+A\left[\operatorname{dn}^{2}(\kappa x \mid m)-E(m) / K(m)\right],
$$

where the (real) parameters $d, A, \kappa$ and $m$ have to be determined from the experimental data, for instance via a least-squares minimization. Note that the approximation (5.3) assumes a wave crest at abscissa $x=0$; if this is not the case, a phase shift parameter has to be introduced and determined from the data. Note also that, with (5.3), the condition (2.4) is fulfilled identically because the squared bracket averages to zero exactly (for all $m$ and $\kappa$ ).

The holomorphic function $\mathfrak{P}$ is then obtained replacing $x$ by $z+\mathrm{i} d$ in (5.3), i.e.

$$
\mathfrak{P}(z)=g d+A\left[\mathrm{dn}^{2}(\kappa(z+\mathrm{i} d) \mid m)-E(m) / K(m)\right],
$$

and the function $\mathfrak{Q}$ is subsequently derived at once substituting (5.4) into (4.1):

$$
\mathfrak{Q}(z)=(A / \kappa)[\mathrm{Z}(\kappa(z+\mathrm{i} d) \mid m)-\mathrm{Z}(\mathrm{i} \kappa(a+d) \mid m)],
$$

where $\mathrm{Z}$ is the Jacobi zeta function (Abramowitz \& Stegun 1965). For $(2 \pi / k)$ periodic waves, we have $\kappa=k K(m) / \pi$, and for solitary waves (i.e. $k \rightarrow 0, m \rightarrow 1$ ) the approximation (5.5) becomes

$$
\mathfrak{Q}(z)=(A / \kappa)[\tanh (\kappa(z+\mathrm{i} d))-\mathrm{i} \tan (\kappa(a+d))] .
$$

This example also shows that the calculation of $\mathfrak{Q}$ is a simple matter.

\subsection{Remarks}

Other basis of functions can be used to represent the pressure at the bottom $\mathfrak{p}$, but the two examples above are a priori the more relevant for practical applications. 


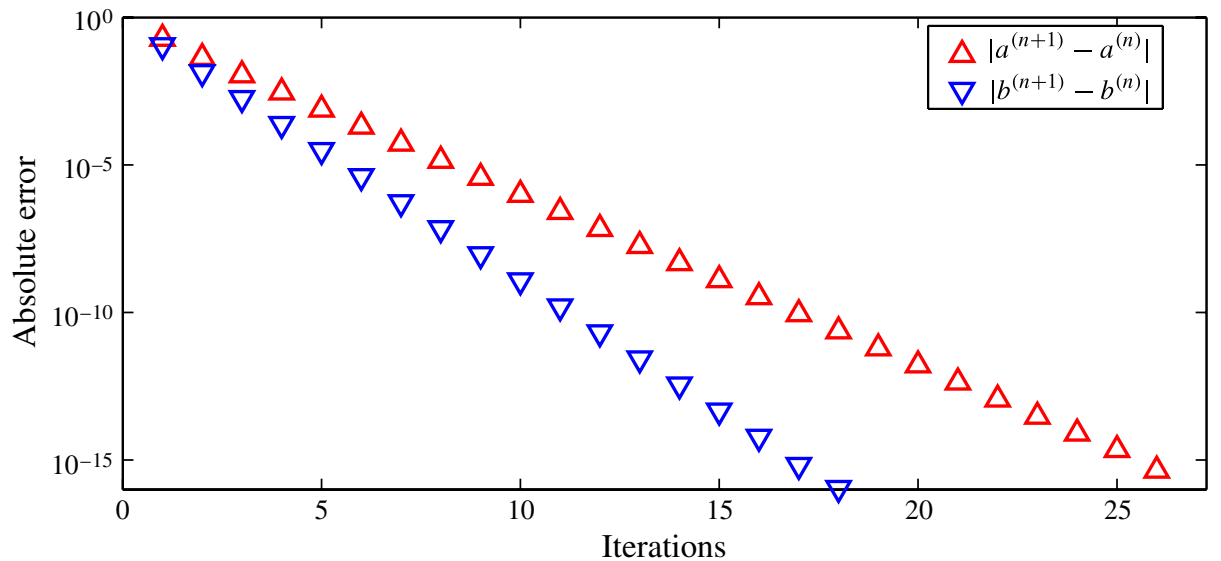

FIGURE 1. (Colour online) Numerical fixed-point iterations of (3.6) (upward triangles) and (3.7) (downward triangles) for the fifth-order Fourier reconstruction.

The convergence of (5.1) as $N \rightarrow \infty$ has already been addressed by Clamond \& Constantin (2013). Their numerical example also shows that this Fourier approximation can be used in numerical applications. Further numerical examples are provided in $\S 7$.

The validity of approximations (5.4)-(5.5) is not limited to shallow water. Indeed, this kind of approximation is quite accurate for all depths, even for waves of large amplitude (Clamond 1999, 2003). Further evidence is given in $\S 7$ below. If more accuracy is required, then higher-order cnoidal-like approximations can be introduced (Fenton 1979; Clamond \& Fructus 2003).

\section{Convergence outline}

A remaining open question is whether or not the iterations of (3.7) and (4.4) converge. Numerical investigations suggest that converge occurs (see §7), this convergence is supported by the theoretical investigation of this section.

Following Clamond \& Constantin (2013), the convergence of (3.7) is investigated introducing the functional iterations

$$
y=F(y) \equiv \frac{\operatorname{Re}\{\mathfrak{P}(\pi / k+\mathrm{i} y)\}}{g}-d=y+\frac{p(\pi / k, y)}{g}, \quad y \in[-d,-b] .
$$

Iterations of (6.1) converge if $F$ is a contraction, i.e. convergence is ensured if $-1<F_{y}<1$, divergence occurs if $\left|F_{y}\right|>1$ and if $\left|F_{y}\right|=1$ iterations may converge or not depending on $F$ (Devaney 1989). For (6.1), convergence is thus guaranteed if $-2 g<p_{y}<0$ for $y \in[-d,-b]$. With the results by Constantin (2006) and Constantin \& Strauss (2010) yielding a rigourous proof of the inequality $p_{y} \leqslant-g$ under the troughs of Stokes waves, the upper bound for contractivity is fulfilled. The lower bound $p_{y}>-2 g$ is obviously satisfied for solitary waves (since then $p_{y} \rightarrow-g$ as $x \rightarrow \pm \infty$ ), but a rigourous proof for general waves is apparently not available in the literature. Numerical experiments (see figure 1 below) suggest that iterations (3.7) converge, even faster than iterations (3.6). 
In order to investigate the convergence of (4.4), let the functional iterations be

$$
y=G(y) \equiv \frac{B}{g}-\frac{\sqrt{(B-g a)^{2}-2 g \operatorname{Im}\{\mathfrak{Q}(x+\mathrm{i} y)\}}}{g}, \quad y \in[-d, \eta(x)],
$$

for a known holomorphic function $\mathfrak{Q}$ at a fixed abscissa $x$ and for given $a$ and $B$. Iterations of (6.2) converge if $G$ is a contraction (i.e. if $-1<G_{y}<1$ ), the definition of $G$ giving

$$
G_{y}=\frac{[\operatorname{Im}\{\mathfrak{Q}\}]_{y}}{\sqrt{(B-g a)^{2}-2 g \operatorname{Im}\{\mathfrak{Q}\}}}=\frac{[\operatorname{Im}\{\mathfrak{Q}\}]_{y}}{B-g y} .
$$

From the definitions (3.1) and (4.1) of, respectively, the functions $\mathfrak{P}$ and $\mathfrak{Q}$, using the Cauchy-Riemann relations and the Bernoulli equation (2.2), we have

$$
[\operatorname{Im}\{\mathfrak{Q}\}]_{y}=\operatorname{Re}\{\mathfrak{P}\}-g d=\frac{1}{2} B-\frac{1}{2} u^{2}+\frac{1}{2} v^{2}=p+g y+v^{2},
$$

so the relation (6.3) can be rewritten

$$
G_{y}=\frac{p+g y+v^{2}}{B-g y}=\frac{p+g y+v^{2}}{2 p+g y+u^{2}+v^{2}} .
$$

The upper bound for contraction condition $G_{y}<1$ yields the inequality $p+u^{2}>0$ that is always satisfied, except at the (angular) crest of the highest wave where $p=u=v=0$ (stagnation point at the crest). Conversely, the lower bound $G_{y}>-1$ yields the inequality $p+v^{2}+B>0$ that is obviously always satisfied. Therefore, $G$ is a contraction everywhere in the bulk and at the free surface and the iterations (6.2) converge to a unique solution (Devaney 1989), except perhaps at stagnation points of the free surface (angular crests forming a $120^{\circ}$ inner angle).

Note that this analysis is valid for all regular waves, periodic or not, with one or more crests and troughs per period, symmetric or not. In other words, this proof is not limited to Stokes waves, unlike the proof for (3.6) and the incomplete proof for (3.7) (although convergences of these iterations likely also occur for more general solutions). Note also that, with $G_{y}$ being independent of $a$ (i.e. independent of the choice of $z_{0}$ in the definition of $\mathfrak{Q}$ ), the convergence proof for the iterations (6.2) is valid wherever $z_{0}$ is chosen in the fluid.

\section{Numerical example}

We illustrate the new reconstruction procedure with the same numerical example used by Clamond \& Constantin (2013). Thus, we consider an exact surface wave with a wave height/depth ratio equal to 0.25 and wavelength/depth ratio equal to 5 (i.e. $a / d \approx 0.25, k a \approx 0.314$ ), which is a wave of significant amplitude in rather deep water. This exact solution is computed numerically solving the Euler equations (Fenton 1988) and the exact pressure at the bed is thus obtained. We take 32 samples of the bottom pressure equally spaced over one wavelength. These are the pressure 'measurements' we use for the reconstruction. We suppose that $\rho$ and $g$ are known parameters, the other parameters having to be computed from the data.

Similarly to the approach of Clamond \& Constantin (2013), we do not make any attempt to introduce sophisticated numerical techniques. We use basic numerical implementation of the analytical formulae given above. 


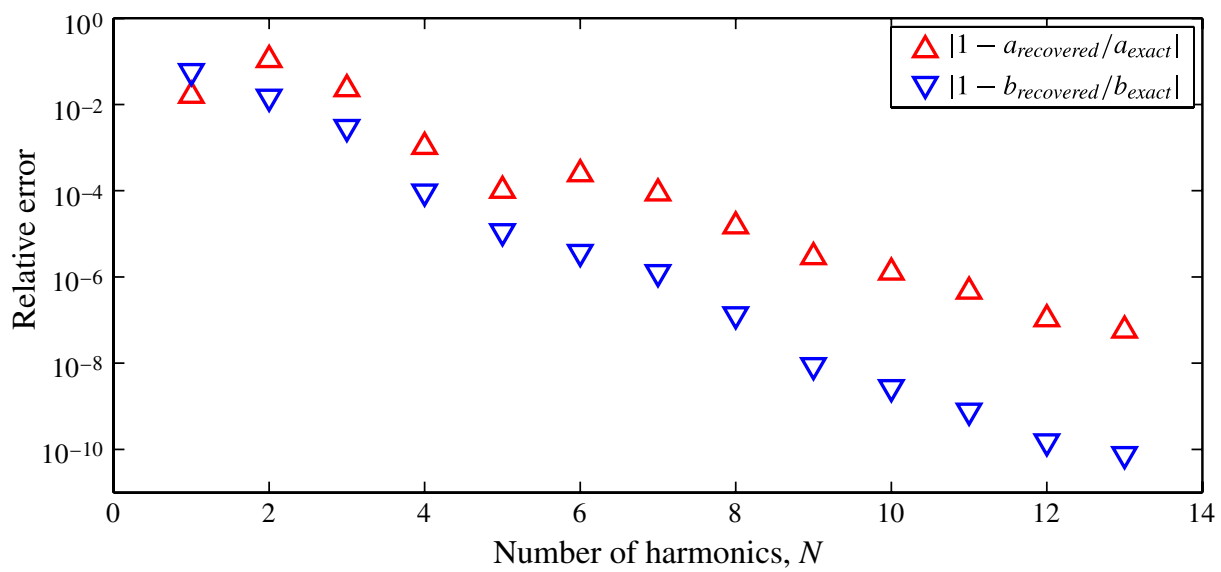

FIGURE 2. (Colour online) Error of the recovered amplitude $a$ (upward triangles) and trough height $b$ (downward triangles) for various-order Fourier reconstructions.

\subsection{Fourier recovery}

An analytic approximation of the pressure at the bottom is obtained by fitting, via least-squares minimization, a fifth-order Fourier polynomial among the pressure data at the bed. The accuracy of this reconstruction is illustrated by Clamond \& Constantin (2013) with various data and graphics. Thus, we focus here on the iterations (3.7) required to compute the trough height $b$.

Starting with initial guess $b^{(0)}=-a$, so that it is guaranteed to lie in the fluid domain, the fixed-point iterations of (3.7) converge, even faster than those of (3.6), as illustrated in figure 1. This result provides numerical evidence of convergence, but a rigourous mathematical proof is still lacking. Note that the rate of convergence is linear here because we use simple fixed-point iterations, but more sophisticated algorithms (e.g. Newton, Halley) will easily provide faster rates of convergence.

For the reconstruction of the whole surface, the formula (4.4) provides the same result (within tiny numerical errors) as the more involved resolution of (3.8) used by Clamond \& Constantin (2013). This is to be expected because (4.4) is an exact solution of (3.8). The speed of the two methods is very different, however.

Using $M=1024$ equally spaced nodes and fast Fourier transforms, the differential equation (3.8) is solved iteratively via a pseudo-spectral method in $O(M \log M)$ operations. This is the fastest possible accurate algorithm. Another resolution of comparable accuracy using, say, a Runge-Kutta method, would be much slower (typically in $O\left(M^{2}\right)$ operations). Conversely, the fixed-point iterations of (4.4) give the same result in $O(M)$ operations. For the example considered here, the surface is obtained about 13 times faster using (4.4) than using (3.8). This gain is consistent with the value $\log _{2}(1024)=10$ (characterizing the different number of operations) and the fact that the Bernoulli constant is implicit using (3.8), thus requiring nested loops to compute the solution (Clamond \& Constantin 2013), while it is explicitly known in the new algorithm.

We also investigated the effect of the number of harmonics $N$ in the reconstruction of the free surface. Figure 2 shows that the accuracy increases with the number of harmonics, and that the trough height $b$ is always a fixed point of (3.7). 


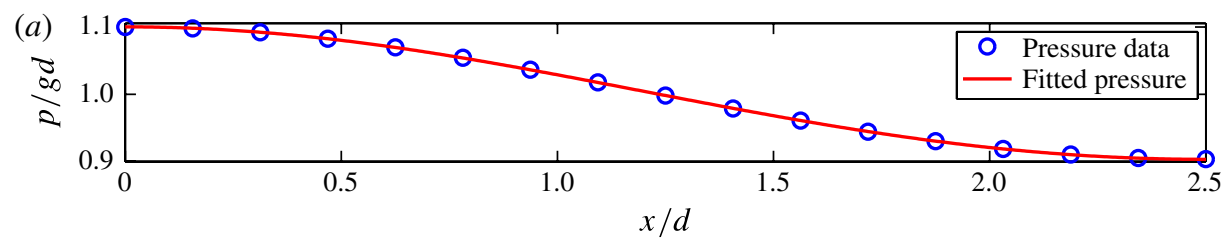

(b) $\quad\left(\times 10^{-4}\right)$

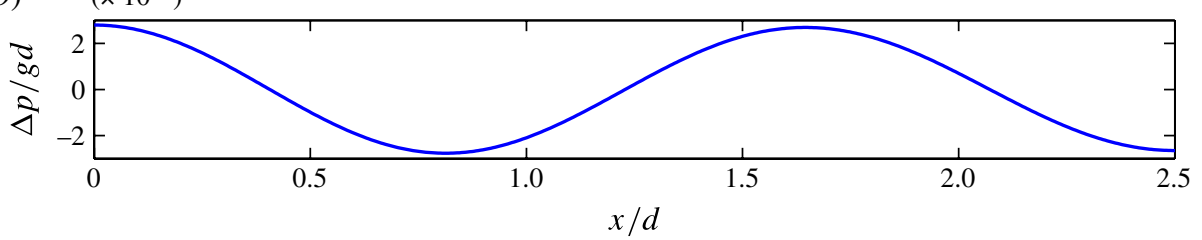

(c)
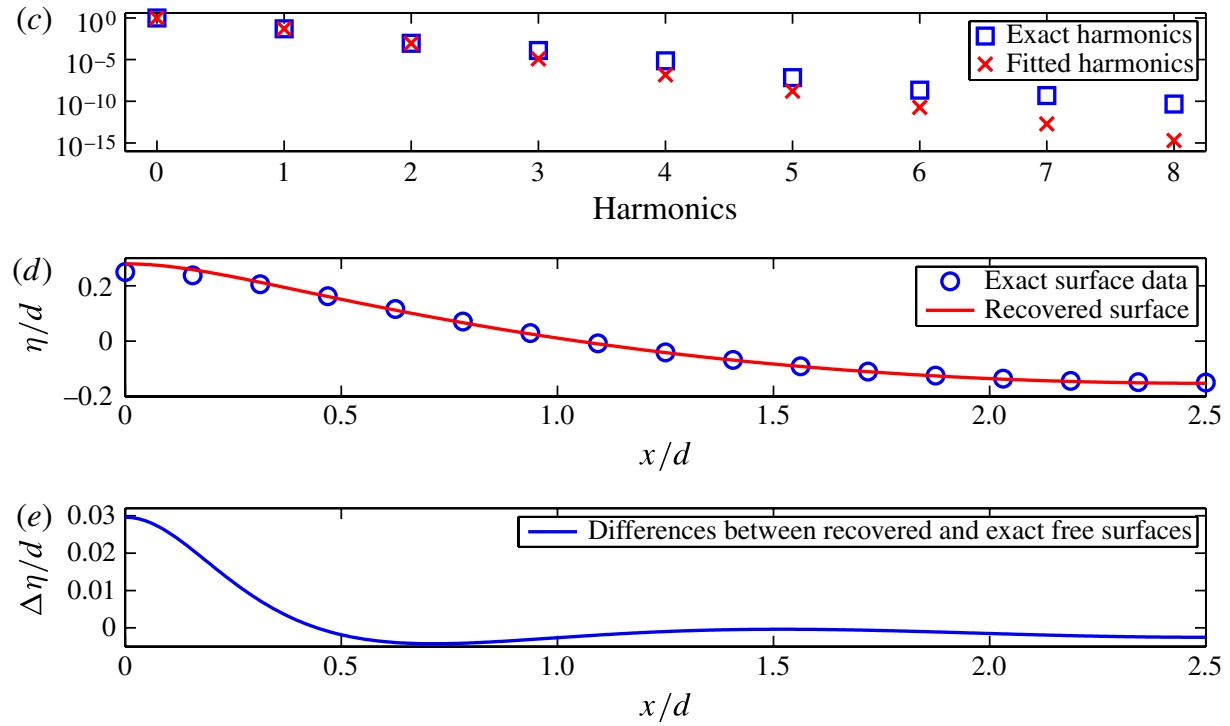

FIGURE 3. (Colour online) Recovery using the cnoidal-like ansatz (5.3): (a) profile of pressure at the bed; $(b)$ difference between fitted and exact pressure at the bed; $(c)$ Fourier spectrum of pressure at the bed; $(d)$ profile of the recovered free surface; $(e)$ recovered free surface error.

\subsection{Cnoidal-like recovery}

We analyse here the relevance of the cnoidal-like ansatz (5.3). It should be emphasized that using this ansatz is a priori difficult because we are dealing with a wave in rather deep water.

The parameters involved in (5.3) being obtained by least-squares minimization, the pressure at the bed (figure $3 a$ ) is well-approximated by the ansatz (5.3), i.e. the accuracy is about four digits (figure $3 b$ ) and the Fourier analysis shows a spectral content matching the exact one (figure $3 c$ ). The reconstructed free surface is reasonably accurate (figure $3 d, e$ ), although not as accurate as the fifth-order Fourier reconstruction.

However, the fifth-order Fourier approximation has more free parameters to be adjusted than the cnoidal-like approximation. For a fair contest, we should compare two ansatz having exactly the same number of free parameters. Therefore, in that 


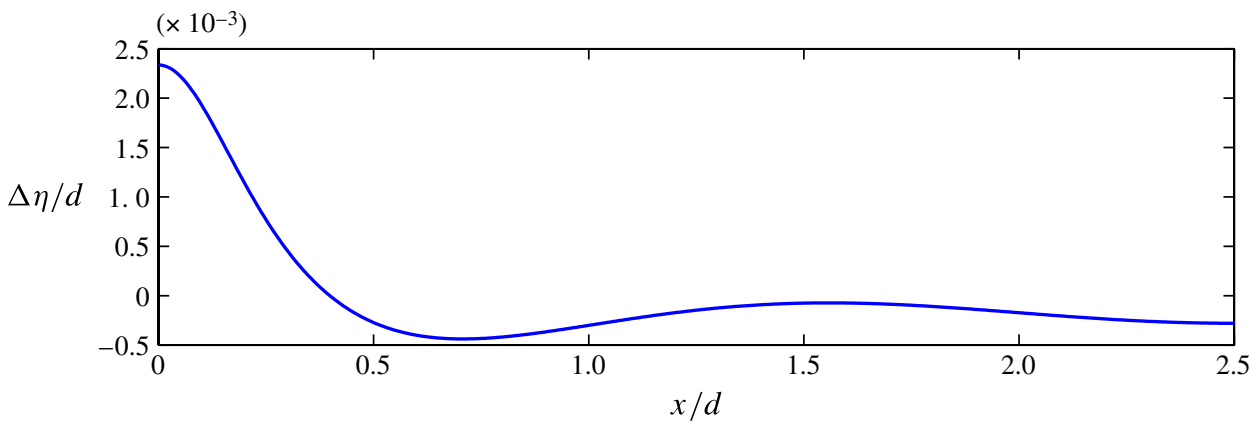

FIGURE 4. (Colour online) Free surface difference between cnoidal-like and second-order Fourier recoveries.

respect, we should compare (5.3) with a second-order Fourier approximation, that is (5.1) with $N=2$. We thus find that these two approximations have comparable accuracies (figure 4).

\section{Conclusion}

We have derived some new exact relations for the reconstruction of the free surface wave profile from pressure measurements at the bed. These new relations allow an easy and fast reconstruction, which is interesting for practical applications. This was possible thanks to the introduction of the holomorphic function $\mathfrak{Q}$. Indeed, the function $\mathfrak{Q}$ yields a reformulation of the problem into a much simplified, but still exact, form. Efficient algorithms and easy proofs then follow at once. It remains to determine whether the present reconstruction procedure works for limiting waves with a stagnation point at the angular crest. This is a problem of more theoretical than practical interest. For practical applications, the method efficiency is illustrated with some numerical examples.

The recovery procedure described here is promising. It would be interesting to see whether a similar approach could be achieved to treat other water wave problems, such as unsteady flows and varying bottom. These matters will be the focus of future investigations.

\section{REFERENCES}

Abramowitz, M. \& Stegun, I. A. 1965 Handbook of Mathematical Functions. Dover.

Bishop, C. T. \& Donelan, M. A. 1987 Measuring waves with pressure transducers. Coast. Engng 11, 309-328.

Clamond, D. 1999 Steady finite amplitude waves on a horizontal seabed of arbitrary depth. J. Fluid Mech. 398, 45-60.

Clamond, D. 2003 Cnoidal-type surface waves in deep water. J. Fluid Mech. 489, 101-120.

Clamond, D. \& Constantin, A. 2013 Recovery of steady periodic wave profiles from pressure measurements at the bed. J. Fluid Mech. 714, 463-475.

Clamond, D. \& Fructus, D. 2003 Accurate simple approximation for the solitary wave. C. R. Mécanique 331, 727-732.

Constantin, A. 2006 The trajectories of particles in Stokes waves. Invent. Math. 166, 523-535.

Constantin, A. 2012 On the recovery of solitary wave profiles from pressure measurements. J. Fluid Mech. 699, 373-384. 
Constantin, A. \& Strauss, W. 2010 Pressure beneath a Stokes wave. Commun. Pure Appl. Maths 63, 533-557.

Deconinck, B., Oliveras, K. L. \& Vasan, V. 2012 Relating the bottom pressure and the surface elevation in the water wave problem. J. Nonlinear Math. Phys. 19 (1), 1240014.

Devaney, R. L. 1989 An Introduction to Chaotic Dynamical Systems, 2nd edn. Addison-Wesley.

Escher, J. \& SChlurmanN, T. 2008 On the recovery of the free surface from the pressure within periodic traveling water waves. J. Nonlinear Math. Phys. 15, 50-57.

FEnton, J. D. 1979 A high-order cnoidal wave theory. J. Fluid Mech. 94, 129-161.

Fenton, J. D. 1988 The numerical solution of steady water wave problems. Comput. Geosci. 14 (3), 357-368.

LONGUET-Higgins, M. S. 1975 Integral properties of periodic gravity waves of finite amplitude. Proc. R. Soc. Lond. A 342, 157-174.

Oliveras, K. L., Vasan, V., Deconinck, B. \& Henderson, D. 2012 Recovering surface elevation from pressure data. SIAM J. Appl. Maths 72, 897-918.

Tsai, C. H., Huang, M. C., Young, F. J., Lin, Y. C. \& LI, H. W. 2005 On the recovery of surface wave by pressure transfer function. Ocean Engng 32, 1247-1259. 